\title{
Topics in statistical mechanics: the $n$-friendly walker model and benzenoid systems
}

\author{
MaRKuS Vöge
}

This thesis consists of two parts. In Part 1 we discuss the so-called $n$-friendly walker model on the directed square lattice. This model generalises the vicious walker model by allowing the walkers to share up to $n$ consecutive lattice sites. We studied two types of configurations that are of particular interest, stars and watermelons, and concentrated on the cases $n=0,1,2$ and configurations consisting of 2,3 and 4 walkers. For two walker stars and watermelons we solved the model for all $n$ and gave the anisotropic generating functions. In the three walker case we studied the isotropic model and conjectured the 1-friendly star generating function and a 1-friendly watermelon recurrence relation. In the anisotropic case we studied configurations with a limited number of steps in one direction. The generating functions for these configurations are rational and we studied their structure. In the case of four walkers we studied the singularities of the isotropic generating function. Furthermore we found a general inversion relation for vicious walker watermelons with arbitrarily many walkers. We conclude this part with an overview of the asymptotics of the generating functions for any number of walkers and for any dimension.

Part 2 deals with benzenoid systems which have an interpretation in the context of chemistry as benzenoid hydrocarbons and in the context of statistical mechanics as self-avoiding polygons enumerated by area on the hexagonal lattice. We also study polyominoes on the hexagonal lattice. We proved that fixed and free polygons (polyominoes) have the same critical point and exponent, and that the amplitudes differ by a factor of 12 . We used the finite lattice method with transfer matrices (FLM/TM) to enumerate benzenoid systems and polyominoes up to area 35. The FLM/TM algorithm employed is exponentially faster than direct enumeration algorithms; it is even exponentially faster that the standard FLM/TM algorithm thanks to a recently developed time and memory saving technique. The complexity of the algorithm employed is $\approx 1.65^{h}$, with $h$ the area of polygons, compared to $2^{2 h / 3} \approx 2.08^{h}$ for the FLM/TM without this

Received 27th October, 2003

Thesis submitted to the University of Melbourne February 2002. Degree approved July 2003. Supervisor: Professor Anthony J. Guttmann.

Copyright Clearance Centre, Inc. Serial-fee code: 0004-9727/04 \$A2.00+0.00. 
improvement and $\kappa^{h} \approx 5.16^{h}$ for direct enumeration algorithms for polygons. We use the enumeration data for benzenoid systems and polyominoes to estimate the growth constants to be $\kappa=5.16193016(8)$ and $\tau=5.1831478(17)$ for polygons and polyominoes respectively. We find strong numerical evidence that the critical exponent is equal to -1 corresponding to a logarithmic singularity in the generating function. We also study the corrections-to-scaling at the critical point. We improved the known rigorous upper and lower bounds significantly to $4.7892 \leqslant \kappa \leqslant 5.9045$ and $4.8049 \leqslant \tau \leqslant 5.9045$. The lower bound used a concatenation argument combined with the enumeration data. We found that two concatenations which were used in the literature to obtain improved lower bounds fail in the cases to which they have been applied. The upper bound required a separate enumeration of objects which over-count polyominoes.

France 